\title{
Self-harm: working with vulnerable adolescents
}

S elf-harm among young people is a major public health issue. Estimates show that as many as 1 in 15 young people self-harm in the UK, which is higher than the rest of Europe (Mental Health Foundation (MHF), 2014). Self-harm results in at least 150000 attendances at accident and emergency departments each year and is one of the top five causes of acute medical admission (Nixon, 2011).

Self-harm is a sign of serious emotional distress (Department of Health (DH), 2014a). Young people can be vulnerable to suicidal feelings, and the risk is greater if they have a previous history of $(\mathrm{DH}, 2011)$ :

\section{$>$ Mental health problems \\ $>$ Behavioural disorders \\ $>$ Substance misuse \\ $>$ Family breakdown \\ Suicide in the family.}

Those in the best position to recognize whether a child or young person is experiencing mental health difficulties include the individual themselves, family members, friends and peers. Workers and professionals in Tier 1 universal services, such as practice nurses, health visitors, school nurses, GPs, teachers and social workers, are the front line of contact. More than any other patient group, children and adolescents have frequent contact with a variety of professionals.

Practice nurses are in a unique position to pick up on behaviour change that may give cause for concern by observing physical signs such as scarring, cuts, bruises or burns. Changes in behaviour can be tricky to interpret when working with this patient group, given that 'risk-taking' is part of the developmental process of adolescent pathology. It is thus important to respond to any intuitive feelings that arise during contact with a young person who could be self-harming, as they are usually founded.

\section{Increasing vigilance}

There is often universal misunderstanding about self-harm by the people closest to the individual, often leading to poor responses
(MHF, 2006). Self-harm continues to be stigmatized, often remaining hidden, which can lead to guilt and shame that is often compounded by the reactions of others (National Society for the Prevention of Cruelty to Children (NSPCC), 2009). The Preventing Suicide in England (DH, 2012) strategy suggests that appropriate training on self-harm and suicide should be available for staff working in these settings. This is a move in the right direction as self-harm is distressing not only for the individual, but also the health professionals involved.

Practice nurses need to be vigilant in recognizing individuals who self-harm and equipped to respond appropriately. The risk of completing a suicide in adolescents who self-harm is 100 times more than that of the general population (National Institute for Health and Care Excellence (NICE), 2002; DH, 2014a). Furthermore, half of the individuals who complete a suicide will have previously engaged in self-harming behaviour, and a quarter in the previous year prior to a suicide completion (NICE, 2002; DH, 2014a). The task therefore is one of knowing how best to respond and support young people who are engaging in self-harming behaviour.

\section{Deliberate self-harm and self-harm}

One of the first indicators of emotional distress is deliberate self-harm and/or selfharming behaviour, and there are increasing reports of significant numbers of young people self-harming in school settings (DH, 2004).

Frequently there is a minimizing of selfharming behaviour among professionals in health care and education settings. Such behaviour is commonly described as 'attention-seeking' and, although there may be some truth in this, it must be remembered that this is occurring alongside a young person in psychological distress. Many young people do quite the opposite of 'seeking attention' and are very secretive and try to conceal their self-harming behaviour.

Deliberate self-harm, self-harm and suicidal behaviour are emotional disorders that exist on a similar continuum as they are all responses to stress. The main difference lies in
Self-harm is one of

the top five causes

of acute medical

admission for

adolescents in the UK.

Maddie Burton

explains how

practice nurses can

recognize and respond

to this vulnerable

patient group

Maddie Burton is senior lecturer in child and adolescent mental health, Institute of Health and Society, University of Worcester

Submitted 17 February 2014; accepted for publication following peer review 2l March 2014

Key words: Deliberate self-harm, suicide, suicide ideation, triggers 
the fact that an individual engaging in suicidal behaviour may wish to die or be ambivalent, while an individual engaging in self-harming behaviour such as cutting may not necessarily have an active wish to die. Self-harm tends to be a way of coping, whereas suicide is more about giving up (Burton et al, 2014).

As opposed to an intention to kill oneself, self-harming is a way in which the young person can get in touch with their psychological turmoil through the physical reality of pain. For the young person, selfharm is perceived as a solution to the problems they are facing. This is why the underlying reasons need to be explored, rather than just focussing on the injuries themselves (NICE, 2004).

It can be difficult to have distinct definitions of self-harm and deliberate self-harm. This is because all self-harming behaviours are relative to the individual, and dependent on how intentional and frequently self-harm occurs (National Child and Adolescent Mental Health Services (CAMHS) Support Service, 2011). Self-harm and deliberate selfharm behaviours can include (Royal College of Psychiatrists, 2012):

\footnotetext{
$>$ Poisoning

Cutting

Excessive alcohol consumption

Illegal drug use

$>$ Hitting or burning oneself.
}

All of these activities have the potential to proceed on a continuum from self-harm to deliberate self-harm, including suicidal behaviour or ideation. Some children may have both a physical illness and mental health problems. For example, a young person with diabetes may place themselves at risk of serious complications through noncompliance with treatment. Thus, it is crucial for the practice nurse to respond appropriately.

\section{Prevalence}

The UK saw an increase of $28.1 \%$ in teenage deliberate self-harm between 1985-1995 (DH, 2004). Up to half of these were likely to have a major depressive disorder and individuals were shown to be at an increased risk of suicide. Suicide was the most frequent cause of death among males aged 15-24 years, and the third most frequent cause of death among females in the same age group.

A survey of 600015 - to 16-year-olds in England found that $6.9 \%$ had engaged in self-harm, with $12.6 \%$ presenting at a hospital for treatment (Hawton et al, 2001). The survey also found that self-harm is more common in females than males, at $11.2 \%$ and $3.2 \%$ respectively. Self-cutting accounted for $64 \%$ of cases, and self-poisoning $30.7 \%$.

Furthermore, a study of 2000 pupils aged 15-16 years from secondary schools in Scotland showed that prevalence of self-harm was similar to that of England (O'Connor et al, 2009). The report identified that girls are three times more likely to report self-harm than boys. The most common motive described was to get relief from 'a terrible state of mind'. Almost 4 in 10 teenagers in the study reported that they 'wanted to die'.

Mental health is understood differently and varies between cultures, although few studies have been undertaken that focus on children and young people and ethnicity. The Chief Medical Officer recommends a repeat of the Office for National Statistics (2004) study Mental Health of Children and Young People in Great Britain, with a particular reference to ethnic minorities, as this is a poorly and patchy researched area (DH, 2013).

\section{Looked-after children}

Adverse and abusive experiences in childhood are associated with an increased risk of suicide (DH, 2011). Looked-after children have an increased risk of self-harming behaviour and mental health problems ( $\mathrm{DH}, 2011)$.

Care-leavers are between four and five times more likely to self-harm in adulthood. They are also at fivefold increased risk of all childhood mental, emotional and behavioural problems, and six to seven times more likely to have conduct disorders (DH, 2013). Hurry and Storey (1998) showed that even though looked after children represent $1 \%$ of the population, they represent $10 \%$ of children and young people presenting to accident and emergency departments following an act of deliberate self-harm.

\section{The developmental process}

Suicide and suicidal ideation always take place in the context of relationships, which are challenging to explore and understand (Hawton et al, 2012). Exceptions are those responding to delusions, or hallucinations linked to drug misuse or psychosis.

The high number of suicide attempts within the adolescent period has links with the developmental process. Adolescence is the most turbulent developmental period since 
infancy, with some of the biggest changes in all three areas of biological, psychological and social change. Risk-taking activity is also part of normal adolescent pathology. This can lead to vulnerability in developing psychosocial disorders, which peak during adolescence (Table 1). These predisposing vulnerabilities can be activated during the adolescent phase (Anderson, 2008).

\section{Physical pain: a medium of communication} During human development, infantile experiences are internalized and attachment patterns laid down, affecting later relationships. Events that trigger self-harming behaviour are action rooted in old patterns and wounds, although these are not necessarily about trauma as not all who selfharm have been victims of abuse or trauma in childhood. The skin becomes a medium for communication (Gardner, 2001)—something is being communicated to others, and it is the health professional's task to interpret and understand this. Very often, the shocking quality communicates the rawness of emotions and impulses and is an essential aspect of self-harming behaviour (Turp, 1999).

It is interesting to consider other powerful body modification similarities where the skin becomes a medium of communication, such as through decoration with tattooing and piercing. Reasons motivating modifications to the external 'skin' or surface are found 'under the skin', within the individual's internal world (Lemma, 2010).

Physical pain is often easier to manage than emotional pain and children and young people who self-harm may find that inflicting pain changes their mood, which can become habit-forming (MHF, 2006). Cutting, for instance, releases endogenous opioids such as endorphins, which produce a brief calming sensation, and serotonin, which lifts the mood (Smith et al, 1998). There is also something about first aid 'patching up' and 'repairing' either by the individual or helpers, with these being experienced as therapeutic.

\section{Triggers}

Bell (2001) argues that the cause given when describing the incident of deliberate self-harm is the 'trigger' which precipitates suicidal behaviour. However, this will often be the reason given by the young person, their family, or even their GP. Common reasons might include an argument with a close friend or failing an exam (Table 2). These can be compounded by adolescent developmental pathology (Beautris 1996; Harrington, 2003).

The notion of a trigger as an explanation, often leads to a minimizing of the level of seriousness surrounding the suicide attempt and is, in practice, never about the stated reason. It is perhaps more of a rationalization of the event rather than an explanation, and it may be a frightening prospect for all concerned to even consider a serious mental disturbance. This is a very important point to bear in mind and is the key to understanding suicidal ideation. Not all individuals who have arguments or fail exams make attempts on their lives. Therefore, those that do so for those reasons given are responding to a trigger (i.e. the argument or exam failure) to much deeper, underlying problems.

The usage of triggers as an explanation can lead to collusion and denial of the seriousness of the event by family members as well as clinical staff, and so it is highly risky not to take the attempt seriously. Suicide attempts must never be minimized by describing somewhat trivial reasons (relative to the self-harm attempt), such as relationship disagreements or exam failure (Burton et al, 2014).

\section{Young people's voices: how can we hear them out?}

The majority of suicide attempters have expressed their thoughts to others, but $75 \%$ of completed suicides have had no contact with mental health services (MHF, 2006). Community settings, including practice nurses, have a vital role in helping adolescents who consider suicide to be an option (DH, 2012). The following testimony was made by a young person in Truth Hurts (MHF, 2006):

Long-term relationships with workers rather than offers of time-limited work provide continuity and the chance to build rapport. I want workers to be more concerned about me genuinely, than only thinking about risks.

Similar wishes were expressed in the Chief Medical Officer's report (DH, 2013):

They want staff who are approachable, available and skilled in engaging and listening to young people. Children and young people valued continuity, confidentiality and support, particularly at transition. Flexibility and accommodation of health workers to the young person's needs were seen as positive attributes.
Table I. Psychosocial disorders that peak during adolescence

Suicide and/or suicide ideation

Self-harm and/or deliberate self-harm

Substance misuse

Offending behaviour

Depression

Eating disorders

Table 2. Potential triggers of self-harm Bullying

Difficulties with parental and peer relationships

Bereavement

Earlier abusive experiences

Difficulties with sexuality

Problems with ethnicity, culture and/or religion

Substance misuse

Low self-esteem

Contextual triggers

Adverse family circumstances

Dysfunctional relationships

Domestic violence

Poverty

Parental criminality

Time in local authority care

Frequent punishments

Family transitions 
One of the transformational aspects of the Children and Young People's Improving Access to Psychological Therapies project (CYP IAPT) (NHS, 2011) is attentiveness to the voices of children and young people. This is in keeping with Article 12 of The United Nations Convention on the Rights of the Child (UNICEF UK, 1989). With CYP IAPT, selfreferral is being implemented, which allows a parent or child to refer themselves into child and adolescent mental health services (CAMHS) without professional referral.

In many areas around the UK, health professionals already have access to local CAMHS. This is an important element, and the NHS is also working in patnership with the education sector to reach out to more children and young people (NHS, 2012). The Royal College of Paediatrics and Child Health has introduced MindEd, an e-resource that provides even wider access to knowledge and information.

\section{Responding to young people who deliberately self-harm}

Skilled support at a first episode of self-harm is evidenced to reduce further self-harm and potentially a suicide attempt (NSPCC, 2009). However, many young people may never have access to specialist services, and may be first noticed in Tier 1 settings by practice nurses. In these cases, it is important to seek advice from CAMH professionals. Very often, CAMHS clinicians will work jointly with staff such as practice nurses, school nurses and counsellors. Professionals and practitioners in Tier 1 universal services need to be making referrals to Tier 2 and 3 CAMHS. They may continue to have ongoing contact with the young person, especially given the increased waiting list times for specialist CAMHS.

Containment and reciprocity are valuable principles for infants, children and young people, no matter which model of intervention is adopted. These principles also form part of the parenting programme, The Solihull Approach (Douglas and Ginty, 2001).

\section{The unique position of the practice nurse}

Practice nurses are in a unique position and often favoured by young people, given their more 'universal' approach. They can be seen and experienced by young people as less stigmatizing than seeing a counsellor, and so offer an important resource.

\section{Making timely referrals}

A practice nurse will need to refer a young person who has engaged in deliberate selfharm to CAMH Tier 2 and 3 services. An accurate assessment and understanding of the young person's internal world is essential in order to initiate management and treatment (Bell, 2001). This would take place if a child or young person had been admitted to a hospital paediatric ward following an act of deliberate self-harm such as an overdose.

Effective assessment and management of self-harm, particularly in emergency departments, is a huge opportunity to reduce repeated episodes of self-harm and future suicide risk (DH, 2014a). Without a thorough psychosocial assessment, one cannot conclude what an intended outcome may have been. NICE (2004) recommends that a specialist assessment should take place within 48 hours of admission to hospital by a CAMHS clinician. The clinician would then make a decision about discharge, both in conjunction with the young person and their family.

A recent study has found that as many as $43 \%$ of young people attending hospital are not receiving a psychosocial assessment as has been recommended. This was despite $84 \%$ of under 16 s being admitted to hospital (Hawton et al, 2012). This is certainly an area for attention as studies with adults have demonstrated that those who received a thorough psychosocial assessment were associated with a $40 \%$ lower risk of repetition (Kapur et al, 2013).

\section{Understanding self-harming behaviour}

Understanding how the young person manages stress is crucial. Self-harming behaviours are evidence of earlier experiences of interpersonal, familial, and relationship difficulties, which lead to insecurity of attachment (Briggs, 2002). Thus, it is not surprising that looked-after children carry an increased risk of self-harming behaviours.

\section{Initiating interventions}

At the heart of any intervention by any health professional is a combination of the personcentred approach with an understanding of the 'systemic' context, which is the system and context surrounding the young person. The person-centred approach emphasizes the therapeutic nature of human understanding, where the self of the therapist and their deep acceptance and respect of the young person as an individual is paramount (Prever, 2010). 
Individual therapeutic work with children and young people rarely involves a sole intervention, and is usually alongside parent and family work. The intervention is always about the appropriate application of a particular model to the young person, and not the other way round (i.e. fitting the young person to the model). This is perhaps coming under increasing pressure as, more than ever before, interventions in CAMHS must be evidence-based best practice (DH, 2013).

Many interventions, despite perhaps being successful, have lacked sufficient supporting research, whereas others are robustly evidenced. This has led to parent training programmes such as Triple P (2014) and cognitive behavioural therapy (CBT) being flagged up as recommended interventions. However, young people have said that CBT, which is now usually offered as standard, does not work for everyone (DH, 2013).

\section{Establishing a positive therapeutic alliance} Although the intervention offered is important, the therapeutic alliance between the clinician and the young person and their family is also a potent determinant of outcomes in mental health. The therapeutic relationship is often more powerful in bringing about change than the actual model of intervention adopted. The particular approach adopted will depend on a variety of factors relating to the individual and their context. For practice nurses, the key points to consider are:

Referral and/or consultation with local CAMHS provision

$>$ Consideration of close or joint working with CAMHS and supervision-this is often a feature of Health Visitor practice

$>$ Acting in the role of containment using a person-centred approach.

Given the likelihood of eventual discharge from CAMHS, it is important to bear in mind the likelihood of a long-term restoration or renewal relationship with the young person. This relationship can act as a protective factor for the young person in the longer term.

\section{Confidentiality and safeguarding}

Caldicott principles govern information sharing in health settings, and can also be extended to social care settings (DH, 1997). The underlying principle is that the sharing of information between professionals is essential

\section{CASE STUDY I}

Sasha, a 15-year-old girl, is in the accident and emergency (A\&E) department following an episode of self-harm. She has consented to the treatment of her physical wounds. As per NICE (2004) guidelines, she has been admitted to the paediatric ward at the hospital to await assessment by Tier 3 child and adolescent mental health services (CAMHS).

This the fourth time in the last 2 months that Sasha has been seen in A\&E for selfharm. She took an overdose of paracetamol the last time. Sasha does not want her parents to know that she self-harms.

Do Sasha's parents need to be notified? According to Gillick competency (British Medical Association, 2001), Sasha is competent and does understand what has happened to her. However, it would not be good practice to accede to Sasha's request. The health professional should apply
Caldicott principles (DH, 1997) and Article 12 from The United Nations Convention on the Rights of the Child (UNICEF UK, 1989).

There may have been a case for not doing so had it been a first admission. However, as this is her fourth admission, Sasha is at a high risk of a completed suicide. Therefore, informing her parents could be justified on the grounds of a risk of significant harm and a threat to her life. Breaching confidentiality would be on the same grounds.

The decision should be made in consultation with Sasha, the A\&E team and the CAMHS clinician conducting the psychosocial assessment. It would not be good practice to see Sasha in isolation of her family, and the wish would be to work alongside all of them. Understanding Sasha and her predicament in the context of her family is the best way to support her and decide what intervention may be required. 
to enable early help for children and young people and to safeguard and promote their welfare (Walker, 2008). There is often a dilemma when a young person discloses selfharm but does not want the information to be shared with their parents (Case Study 1). This could be a young person presenting in primary care to a practice nurse, perhaps with self-inflicted injuries.

\section{Shared decision-making}

Decisions should always be made on a case by case basis and in consultation with the young person and, ideally, their family. Dealing with disclosure requires core skills and awareness of one's own personal feelings, which also includes being able to signpost to services a young person wants.

It is important for the health professional not to work in isolation, but to be supported by colleagues and through supervision. At times, information will have to be shared with professionals working together to ensure that children and young people get the help they need (Department for Education, 2013). Practice nurses have a responsibility to share concerns with the lead for child protection within their organization or children's social care, especially if a young person's selfharming places them at risk of significant harm. This also occurs where it is in the public interest to do so and if a decision needs to be made about sharing information with family and friends (National CAMHS Support Service, 2011; DH, 2014b).

\section{Conclusions}

Uniquely, children and young people will come into contact with a variety of professionals at various points in their lives. It is of great value and advantage for those professionals in front line settings to have knowledge and understanding of child and adolescent mental health issues, although many feel ill-equipped to deal with these ever increasing issues. Practice nurses are often perceived by children and young people as less stigmatizing, and so offer an important window of opportunity for help-seeking and signposting for individuals and their families.

Conflict of interest: none declared.

\section{References}

Anderson R (2008) A psychoanalytical approach to suicide in adolescents. In: Briggs S, Lemma A,

Crouch W, eds. Relating to Self-harm and Suicide. Routledge, Sussex: 61-71

Beautrais A (1996) Serious suicide attempts in young people: a case control study. Thesis. University of Otago, Dunedin

Bell D (2001) Who is killing what or whom? Some notes on the internal phemenology of suicide. Psychoanal Psychother 15(1): 21-37. doi: $10.1080 / 02668730100700021$

Briggs S (2002) Working with the risk of suicide in young people. J Soc Work Pract 16(2): 135-48

British Medical Association (2001) Consent, Rights and Choices in Health Care for Children and Young People. BMJ Publishing Group, London

Burton M, Pavord E, Williams B (2014) An Introduction to Child and Adolescent Mental Health. SAGE, London

Department for Education (2013) Working together to safeguard children: a guide to inter-agency working to safeguard and promote the welfare of children. http://media.education.gov.uk/assets/files/pdf/w/ working\%20together.pdf (accessed 16 April 2014)

Department of Health (1997) Report on the review of patient-identifiable information. http://www.wales. nhs.uk/sites3/Documents/950/DH_4068404.pdf (accessed 17 April 2014)

Department of Health (2003) Confidentiality: NHS code of practice. https://www.gov.uk/government/ uploads/system/uploads/attachment_data/ file/200146/Confidentiality_-_NHS_Code_of_ Practice.pdf (accessed 16 April 2014)

Department of Health (2004) CAMHS standard, national service framework for children, young people and maternity services. http://tinyurl.com/ q59thhd (accessed 24 March 2014)

Department of Health (2011) Consultation on preventing suicide in England: a cross-government outcomes strategy to save lives. http://tinyurl.com/ pxt295u (accessed 20 March 2014)

Department of Health (2012) Preventing Suicide in England: One Year On. https://www.gov.uk/government/uploads/system/uploads/attachment_data/ file/278119/Annual_Report_FINAL_revised.pdf (accessed 24 March 2014)

Department of Health (2013) Annual report of the Chief Medical Officer. Our children deserve better: prevention pays. https://www.gov.uk/government/ uploads/system/uploads/attachment_data/ file/255237/2901304_CMO_complete_low_res_ accessible.pdf (accessed 17 April 2014)

Department of Health (2014a) Preventing suicide in England: one year on. First annual report on the cross-government outcomes strategy to save lives. https://www.gov.uk/government/uploads/system/ uploads/attachment_data/file/278119/Annual Report_FINAL_revised.pdf (accessed 20 March 2014)

Department of Health (2014b) Information sharing and suicide prevention. https://www.gov.uk/government/uploads/system/uploads/attachment_data/ file/271792/Consensus_statement_on_information sharing.pdf (accessed 16 April 2014)

Douglas H, Ginty M (2001) The Solihull Approach: changes in health visiting practice. Community Practitioner 74(6): 222-4

Gardner F (2001) Self-Harm: A Psychotherapeutic Approach. Routledge, East Sussex

Harrington R (2003) Depression and suicidal behaviour. In: Skuse DH, ed. Child Psychology and Psychiatry: An Introduction. The Medicine Publishing Company, Oxford: 125-8

Hawton K, Townsend E, Appleby L et al (2001) Effects of legislation restricting pack sizes of paracetamol and salicylate on self-poisoning in the UK: before and after study. BMJ 2001: 322. doi: $10.1136 /$ bmj.322.7296.1203

Hawton K, Bergen H, Waters K et al (2012) 
Epidemiology and nature of self-harm in children and adolescents: findings from the multicentre study of self-harm in England. Eur Child Adolesc Psychiatry 21(7): 369-77. doi: 10.1007/s00787-012-0269-6

Hurry J, Storey P (1998) Deliberate self harm among young people. http://www.ioe.ac.uk/tcru/pub-ib-7. htm (accessed 20 March 2014)

Kapur N, Steeg S, Webb R et al (2013) Does clinical management improve outcomes following selfharm? Results from the multicentre study of selfharm in England. PLoS One 8(8): e70434. doi: 10.1371/journal.pone.0070434

Lemma A (2010) Under the Skin: A Psychoanalytic Study of Body Modification. Routledge, London

Mental Health Foundation (2006) Truth hurts-report of the national inquiry into self harm among young people. http://www.mentalhealth.org.uk/content/ as sets/PDF/publications/truth_hurts. pdf? view=Standard (accessed 24 March 2014)

Mental Health Foundation (2014) Self-harm. http:// www.mentalhealth.org.uk/help-information/mental-health-a-z/S/self-harm/ (accessed 16 April 2014)

National Child and Adolescent Mental Health Services Support Service (2011) Self-harm in children and young people handbook. http://www.chimat.org. uk/resource/item.aspx?RID=105602 (accessed 16 April 2014)

NHS (2011) Children and young people's improving access to psychological therapies. http://tinyurl.com/ mrjjzoy (accessed 16 April 2014)

NHS (2012) Children and young people's improving access to psychological therapies. Newsletter, December. http://www.iapt.nhs.uk/silo/files/cypiapt-newsletter-3-december-2012.pdf (accessed 16 April 2014)

National Institute for Health and Care Excellence (2002) Scope. http://www.nice.org.uk/nicemedia/ pdf/Self-HarmScopeFinalV3140502.pdf (accessed 24 March 2014)

National Institute for Health and Care Excellence (2004) The short-term physical and psychological management and secondary prevention of self-harm in primary and secondary care. http://www.nice.org. uk/nicemedia/live/10946/29424/29424.pdf (accessed 17 April 2014)

National Society for the Prevention of Cruelty to Children (2009) Young people who self-harm: implications for public health practitioners. http:// www.nspcc.org.uk/Inform/research/briefings/ youngpeoplewhoselfharmpdf_wdf63294.pdf (accessed 24 March 2014)

Nixon B (2011) Self-harm in children and young people handbook. http://www.chimat.org.uk/resource/ item.aspx?RID=105602 (accessed 20 March 2014)

O'Connor RC, Rasmussen S, Miles J et al (2009) Selfharm in adolescents: self-report survey in schools in Scotland. $\mathrm{Br} J$ Psychiatry 194(1): 68-72. doi: 10.1192/bjp.bp.107.047704.

Office for National Statistics (2004) Mental Health of Children and Young People in Great Britain, 2004. http://www.esds.ac.uk/doc/5269/mrdoc/ pdf/5269technicalreport.pdf (accessed 22 April 2014)

Prever M (2010) Counselling and Supporting Children and Young People: A Person-centred Approach. SAGE, London

Royal College of Psychiatrists (2012) Self-harm in young people: information for parents, carers and anyone who works with young people. http://www. rcpsych.ac.uk/healthadvice/parentsandyouthinfo/ parentscarers/self-harm.aspx (accessed 16 April 2014)

Smith G, Cox D, Saradjian J (1998) Women and selfharm. Women's Press, London

Triple P (2014) Where it began. http://www.triplepparenting.net/glo-en/about-triple-p/where-it-began/ (accessed 16 April 2014)

Turp M (1999) Encountering Self-Harm in Psychotherapy and Counselling Practice. British Journal of Psychotherapy 15(3): 306-21

UNICEF UK (1989) The United Nations Convention on the Rights of the Child. http://www.unicef.org. uk/Documents/Publication-pdfs/UNCRC PRESS200910web.pdf (accessed 16 April 2014)

Walker G (2008) Working Together for Children: A Critical Introduction to Multi-agency Working. Continuum, London

\section{Further guidance}

MindEd is an online portal for health professionals who want to identify and understand children and young people with mental health issues. Practical e-learning sessions are available from: www.rcpch.ac.uk/minded

\section{KEY POINTS}

Self-harm among young people is a major public health issue

Practice nurses are in a unique position to pick up on behaviour change that may give cause for concern by observing physical signs

Practice nurses are often perceived by children and young people as less stigmatizing and so offer an important window of opportunity for help-seeking and signposting 